PROBLEMES DE STABILIPE NUMERIQUE POSES PAR LES

SYSTEMES EYPERBOLIQUES AVEC CONDITIONS AUX LIMITES

\title{
J.J. SMOLDEREN
}

Directeur de I'Institut von Karman

Professeur à I'Université de Liège

\section{Introduction}

Un certain nombre de questions importantes de mécanique des fluides font intervenir des équations et systèmes aux dérivées partielles de type hyperbolique. Citons, en particulier, les écoulements instationnaires de fluides compressibles non visqueux, et les écoulements supersoniques stationnaires. Il faut signaler ausi que le traitement numérique de problèmes stationnaires complexes nécessite souvent une approche évolutive, naturelle ou artificielle, dans laquelle l'êtat stationnaire est obtenu comme limite d'une évolution instationnaire à partir de conditions initiales choisies plus ou moins arbitrairement. En l'absence d'ef'ets aissipatifs, une telle approche conduit également à des systèmes différentiels hyperboliques.

La plupart des problèmes hyperboliques rencontrés en mécanique des fluides font intervenir des conditions aux limites imposés aux frontières du champ a'écoulement. Par exemple, Ia condition d'annullation de la composante normale de la vitesse, imposée en chaque point d'une paroi solide.

Dans le cas des écoulements supersoniques stationnaires, il est généralement possible d'identifiex des conditions d'entrée ou d"amont, qui jouent le même rôle que les conditions initiales de problèmes instationaires, et des conditions latérales, souvent imposées sur des lignes de courant, qui peuvent être assimilées aux conditions aux limites de ces problemes.

Ia solution de systèmes hyperboliques par la méthode des différences finies peut être effectuée explicitement, en progressant de proche en proche suivant la variable temporelle dans les problèmes instationnaires, ou suivant une variable spatiale convenablement choisie dans le problème supersonique stationnaire. Les méthodes partiellement ou totalement implicites, qui nécessitent à chaque étape, la solution d'un grand système algébrique a'équations couplées, ne seront pas abordées dans le présent travail.

Il est connu que la methode de progression explicite conduit souvent $\ddot{a}$ des instabilités numériques. II est donc très utile, en pratique, de aisposer d'un critère permettant de vérifier si un schéma 
aux différences finies sera stable ou non. Lorsqu'il s'agit d'équations ou de systèmes aux dérivés partielles lineaires à coefficients constants, traitées par un millage uniforme et en l'absence de conditions aux limites, la question est résolue par la condition nécessaire classique de von Neuman (Ref. l), concernant le module des valeurs propres de la matrice d'amplification de modes de Fourier numériques. Un certain nombre de conditions suffisantes ont été établies (Ref. 2) et Kreiss a étendu la théorie de la stabilité aux systèmes linéaires ä coefficients variables (Ref. 3 ). Les problèmes non liné aires sont évidemment beaucoup plus ardus et l'étude de leur stabilité ne peut être abordée qu'après une linéarisation par rapport à de petites perturbations.

Le critère classique de von Neumann ne s'applique pas, en général, aux problèmes qui font intervenir des conditions aux limites, parce que les modes de Fourier considérés sont le plus sourent incompatibles avec ces conditions.

De nombreux exemples ont d'ailleurs illustré le fait que des instabilités peuvent se manifester dans le traitement de tels problèmes, même si le schéma aux différences utilisé satisfait largement le critère de von Neumann, confirmant ainsi le point de vue adopté par Moretti (Ref. 4) au cours d'une longue controverse au sujet d'instabilités rencontrées dans le traitement des équations de la mécanique des fluides.

La présente note est consacrée a cet aspect de la tiéorie de la stabilicé numérique. La variété des types d'instabilité induites par la présence de conditions aux limites sera illustrée par des exemples et une importante conaition nécessaire de stabilité sera examinee d'un point de vue élémentaire, sans prétendre à la rieueur mathematique. Ces résultats peuvent être d'ailleurs justifiés rigoureusement en faisant appel à la théorie de Godunov et Ryabenkii (Refs. 5 et 6).

Il est important de réaliser qu'un calcul explicite, de proche en proche, nécessite souvent des conditions aux limites plus nombreuses que le problème physique considéré ou que le système aux dérivées partielies qui le représente. En effet, I'utilisation de schémas de discrétisation d'ordre de précision supérieur au premier pour le traitement des dérivées spatiales, nécessitera toujours l'introduction de conditions aux limites aditionnelles de nature essentiellement numérique. Le choix de ces conditions reste arbitraire dans une large mesure, même si l'on satisfait aux exigences de la précision. Malheureusement, un choix apparement raisonnable peut con- 
duire à des instabilités imprévues, si courantes en anelyse numérique.

D'autre part, de nombreux problèmes de mécanique des fluides tels que les écoulements en conduites et les écoulements en champ infini, requièrent l'introduction de conditions d'entrée et de sortie ou de conditions représentant le champ à grande distance. De telles conditions, qui représentent, dans beaucoup de cas, des approximations plus ou moins grossières, ne sont pas entièrement définies par des considérations physiques et leur choix reste donc également arbitraire, dans une certaine mesure. Il faut donc s'attendre à l'apparition de problèmes de stabilité analogues à ceux qui sont introduits par les conditions aditionnelles.

L'utilité a'un critère de stabilité permettant d'orienter le choix des conditions additionnelles et des conditions à l'entrée, à la sortie et au large, est donc évidente.

L'intêrêt d'un tel critère ne se limite d'ailleurs pas au choix de conditions aux limites numériques ou mal définies, comme le montre l'exemple du paragraphe 4, où des conditions aux limites naturelles créent des instabilités, le schéma utilisé étant au premier ordre et stable au sens de von Neumann.

\section{Stabilité numérique en présence de conditions aux limites}

Les aspects essentiels du problème de la stabilité du traitement numérique des problèmes aux limites pour les équations et systèmes hyperboliques linéaires à coefficients constants, peuvent être mis en évidence en traitant le cas le plus simple d'une équation à une seule fonction inconnue de deux variables indépendantes $t, x$. Le domaine consiáéré sera défini par
$t>t_{0}$
$x_{0}<x<x$

et un maillage uniforme sera utilisé, les points mailles étant

$t_{k}=t_{0}+k \Delta t \quad(k=0,1,2, \ldots)$ et $x_{j}=x_{0}+j \Delta x(j=0,1,2, \ldots, N)$

${ }_{j}^{k}$ désignera la valeur approchée de la fonction inconnue au point $\left(t_{k}, x_{j}\right)$ du mailiage.

Des conditions initiales seront donnees en $t=t_{0}$, ce qui se traduira, génêralement, dans la version discrète, par un certain nom- 
bre de relations liant les valeurs de $u_{j}^{k}$ pour $k=0,-1,-2$, etc.

Des conditions aux limites seront imposées soit pour $x=x_{0}$. soit pour $x=X$ et même, dans le cas géréral, en ces deux points frontières.

L'analyse classique de von Neumann est basée sur l'étude de solutions particulières de l'équation discrétisée à variables discretes séparées, du type

$u_{j}^{k}=($ const $) \rho^{k} \lambda^{j}$

Les modes de fourier discrets analogues des composantes de Fourier d'une fonction d'une variable continue, s'obtiennent en remplacant $\lambda$ par les exponentielles complexes

$\lambda_{n}=\exp \left(\frac{i \pi n}{N}\right) \quad(n=0,1,2, \ldots, N)$

Il en résulte un système complet de fonctions discrètes pour le maillage spatial considéré.

Pour qu'une expression, à variebles séparées, du type (2.1) soit solution de l'équation linéaire aux différences finies, obtenue par discrétisation de l'équation hyperbolique proposée, o et $\lambda$ doivent satisfaire une relation algébrique :

$F(0, \lambda)=0$

Cette relation carectéristique du schéma de discrétisation s'obtient en substituant $1^{\prime e x p r e s s i o n ~(2.1) ~ d a n s ~ l ' e ́ q u a t i o n ~ a u x ~ d i f f e ́ r e n c e s ~ f i-~}$ nies (après élimination a'un facteur commun $\rho^{k} \lambda^{j}$ ).

Le critère de von Neumann s'obtient en remplaçant $\lambda$ par les valeurs (2.2) dans cette relation et en exprimant que toutes les valeurs correspondantes de l'amplification temporelle $p$ ont un module inférieur à $\{1+o(\Delta t)\}$.

Ce critère n'est cependant pas géneralement applicable, en présence de conditions aux limites, car les modes de Fourier (2.1). (2.2) ne sont compatibles quave des conditions aux limites homogènes très particulières.

Nous devons donc rechercher d'autres familles, aussi complètes que possible, de solutions simples compatibles avec les conditions aux limites homogènes du problème et étudier leur comportement, borné ou divergent, en fonction de la variable discrète k qui définit l'evolution du calcul. 
Nous n'envisageons que les conditions aux limites qui peuvent s'exprimer par des relations linéaires et homogènes, à coefficients constants, liant les valeurs de u en quelques points du maillage voisins de la frontière. A la frontière $x=x_{0}$, ces relations seront de la forme générale

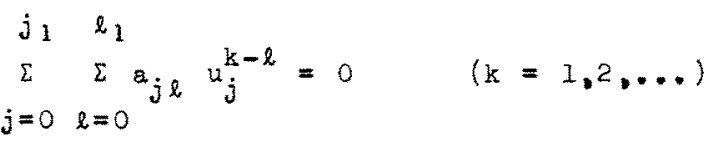

Les coefficients $a_{j \ell}$ sont donnés et $j_{I}, \ell_{1}$ représentent des entiers dépendant de l'ordre de l'équation différentielle étudiée.et de l'ordre de précision de la discrétisation.

Les relations imposées $\grave{a}$ la frontière $x=X$ seront de type analogue :

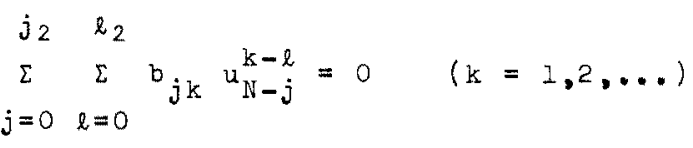

Il est évidement toujours possible de combiner des solutions a variables separees, de type (2.1), correspondant à la même valeur de $\rho$, de façon $\dot{a}$ satisfaire certaines relations du type (2.4) ou $(2.5)$, pour toutes valeurs de $k$.

En effet, la relation caractéristique (2.3) considérée, pour $\rho$ fixé, comme une équation algebrique en $\lambda$, possèdera un certain nombre de racines $\lambda_{1}, \lambda_{2}, \ldots, \lambda_{r}, r$ étant le degré de l'équation (2.3) par rapport à $\lambda$. Ces racines seront, en général, des fonctions du paramètre 0 . (Nous n'examinexons pas le cas des racines multiples). Dans le cas simple examiné ici, r sera le produit de l'ordre de l'équation hyperbolique par l'ordre de précision de la discretisation utilisée.

Une combinaison de la forme

$u_{j}^{k}=\rho^{k}\left(A_{1} \lambda_{1}^{j}+\ldots+A_{f} \lambda_{r}^{j}\right)$

sera solution de l'équation aux différences finies, et la substitution de cette expression dans des conditions de la forme (2.4), (2.5) fournira une relation entre les constantes arbitraires $A$.

Le processus numérique ne sera done possible et déterminé que si le nombre de conditions aux limites (2.4), (2.5) est égal à $r$. En particulier, ce nombre devra être supérieur à l'ordre de l'équation, si la discrétisation utilisée possède une précision a'ordre su- 
périeur è l'unité.

Admettant que nous ayons ainsi choisi $r$ conditions eux Iimites adéquates, l'expression (2.6) sera solution de notre problème homogène, à condition que les $r$ constantes A satisfassent un système algébrique de $r$ équations linéaires homogènes. Une solution non triviale n'existera donc que si le déterminant du systène est nul, condition qui fournit une relation algébrique liant $\rho, \lambda_{1}, \ldots \lambda_{r}$ or les $\lambda_{1}$. $\ldots . \lambda_{r}$. racines de l'équation (2.3) sont des fonctions algébriques, généralement implicites de $p$, de sorte que la condition de compatioilité peut être ramenée à une équation algébrique, généralement très complexe, en $\rho$. Les racines $o_{v}$ de cette équation peuvent être considérées comme des valeurs propres pour notre problème homogène. Les valeurs correspondantes de $\lambda: \lambda_{\nu_{1}}, \ldots \lambda_{\nu r}$ permettront alors de construire des solutions de type $(2.6)$ compatibles avec les $r$ conditions aux limites (noturelles et additionnelles).

Une condition nécessaire de stabilité tout è fait analogue à celle de von Neumann peut donc en être immédiatement déduite, en exprimant tout simplement que les amplifications temporelles $\rho_{v}$ des modes compatibles possèdent un module inférieur à $\{1+\theta(\Delta t)\}$.

En géneral. les modules des valeurs correspondantes $\lambda$ vq $(q=1 \ldots r)$ de $\lambda$ seront différentes de l'unité, de sorte que le nouveau critère de stabilité ne pourra être entièrement équivalent au critère de von Neumann. En fait, il est souvent plus restrictif comme le montreront les considérations et les exemples qui suivent.

L'appication concrète du cxitère présenté parsît cependant conduire ¿̀ des difficultés algébriques pratiquement insurmontables. En effet. I'existence simultanée de conditions aux limites en $x_{1}=x_{0}$ et en $x=X$ introduit nécessairement des temes en $\lambda_{v q}^{\mathbb{N}}$ dans le déterminant au système algébrique des constantes A. L'équation aux valeurs propres ov possèdera donc un degré croissant très rapidement avec $N$. nombre qui est, en principe, très éleve. Une étude asynptotique, pour $N$ tendant vers l'infini, semble doncen particulier, présenter des difficultes transcendantes.

Les développements qui vont suivre tendent à montrer que la situation n'est pas aussi inextricable, grâce à une propriété fondamentale, en vertu de laquelle le nombre des modes compatibles instables éventuels est toujours limité, quel que soit le nombre $\mathbb{N}$ des mailles. 


\section{Modes instables de frontière}

Les modes compatibles avec les conditions aux limites forment une famille analogue à celle des modes de Fourier et qui comporte le même nombre d'éléments, nombre qui tend d'ailleurs vers l'infi$\mathrm{ni}$ avec le nombre de mailles $N$. Les propriétés de cette nouvelle famille sont cependant beaucoup plus complexes que celles des modes de Fourier ortho-normés. En particulier. les coefficients de la représentation de conditions initiales quelconques comme combinaison linéaire de modes compatibles, peuvent présenter des comportements anormaux lorsque N tend vers l'infini. L'utilisation de ces modes en vue de la représentation générale des solutions des équations aux différences finies est donc peu commode.

Dans I'étude de la stabilité, nous pouvons cependant nous limiter à l'examen des modes instables éventuels $(|\rho|>I+\theta(\Delta t))$ et nous allons étabiir que le nombre de tels modes est toujours borné, quel que soit le nombre des mailles $\mathbb{N}$ à condition que le critère de von Neumann soit satisfait. Cette propriété fondamentale, qui simplifie considérablement l'analyse de la stabilité en présence de conditions aux limites, découle d'un découplage remarquable qui se manifeste, pour $\mathbb{N} \rightarrow \infty$, entre les influences des conditions aux limites appliquées aux différentes frontières.

Nous allons tenter de donner une justification plausible à ces conclusions, sans entrer dans les détails algébriques.

Remarquons, tout d'ebord, que la relation caractéristique (2.3) fait correspondanre à chaque point du plan complexe $\lambda$, un certain nombre de points du plan complexe $\rho$. Le critère de von Neumann peut être interprété de la façon suivante: les images des points du cercle unitaire $|\lambda|=1$, lieu des points représentatifs des modes de Fourier spatiaux, doit nécessairement être contenu (à la limite $\Delta t \rightarrow 0$ ), dans le disque unitaire $|\rho| \leq 1$ du plan $\rho$.

Les $r$ valeurs de $\lambda$ correspondant $\hat{a}$ un mode instable $(|p|>1)$ ne peuvent doncêtre de module unitaire, ce qui permet de les classer en deux groupes suivant que leur module est inférieur ou supérieur à l'unité :

$\left|\lambda_{i}\right|<1$ pour $i=1, \ldots, s ; \quad\left|\lambda_{i}\right|>1$ pour $i=s+1, \ldots, r$.

Il est facile de voir que le nombre $s$ des $\lambda$ de module inférieur à I'unité est indépendant de $\rho$, pour $|\rho|>1$. En effet, ce nombre ne peut varier que si un point représentatif de $\rho$ traverse l'image du cercle 
unitaire $|\lambda|=1$, ce qui est impossible tant que le module de o reste supérieur à Irunité, en vertu du critère de von Neumann.

Cette remarque nous permet d'évaluer le nombre $s$ dans un cas particulier, et le passage à la limite $|\rho|+\infty$ conduit le plus rapidement au resultat.

En effet. la relation caracteristique (2.3) (non réduite au même dénominateur) comporte des termes en o et en les puissances de $\frac{1}{p}$. et une serrie de termes faisant intervenir des puissances positives de $\lambda$ et de $\frac{\lambda}{\lambda}$. Lorsque $\rho$ tendra vers l'infini, certaines racines $\lambda$ tendront vers l'infini et $a^{\prime}$ autres vers zếro. Ce sont ces dernières qui nous intéressent car leur module sera nécessairement inférieur à I'unité. La multiplicité de ces racines est évidemment égale à la puissance la plus élevee de $\frac{1}{\lambda}$ qui apparait dans la rejation caractéristique non réduite.

Or cette puissance représente le nombre d'indices inférieurs à j apparaissant dans l'équation aux différences finies correspondant a la maille $(k, j)$. Cette équation fait donc intervenir les valeurs $u_{j-s}, u_{j-s+1}, \ldots, u_{j}, u_{j+1}$ etc.

Il est clair que le rombre de conditions requises à la frontière $\mathrm{x}=\mathrm{x}_{0}$ pour determiner le processus $\mathrm{n}$ uérique sera égal à $\mathrm{s}$. llous pouvons donc conclure que pour chaque valeur de o de module superieur a l'unjté. qui pourrait fournir éventuellement un mode instable, correspondent des valeurs de $\lambda$ de module inférieur à l'unité en nombre égal au nombre $s$ de conditions a imposer en $x=x_{0}$ et des valeurs de $\lambda$ de module supérieur è l'unité dont le nombre r-s est égal à celui des conditions à imposer en $x=x$.

Essayons maintenant d'imaginer la configuration du déterminant du système linéaire de $r$ équations à $r$ inconnues $A_{1}, \ldots$ A $r$ qui s'obtient en écrivant que le mode considéré (2.) satisfait les $r$ conditions aux limites. Ce déterminant peut être décomposé suivant le schéma suivant : 


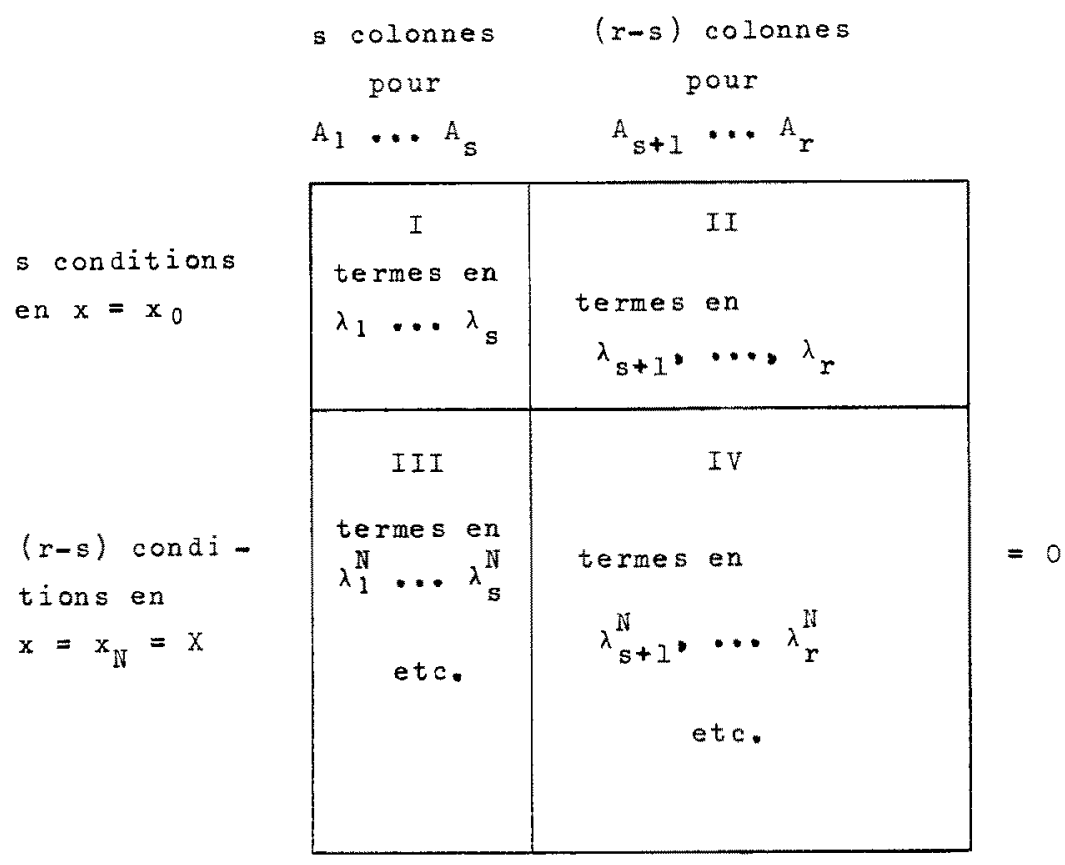

$0 \quad(3.1)$

Lorsque le nombre de mailles tendra vers l'infini, les termes du bloc (IV) domineront tous les autres, puisque les modules des $\lambda_{s+I}, \cdots, \lambda_{r}$ sont supérieurs à I'unité. Si nous développons le déterminant par la règle des mineurs associés, en utilisant les déterminants $(s \times s)$ formés à partir des premières colonnes et des déterminants $(r-s) \times(r-s)$ formés à partir des $r-s$ des autres colonnes, le terme principal sera évidemment

$\{$ déterminant (I) $\} \times\{$ déterminant (IV) $\}$

Tous les autres produits seront négligeables par rapport à ce terme, pour $\mathbb{N} \rightarrow \infty$, car ils feront intervenir un déterminant $(r-s) \times(r-s)$ qui comportera moins de r-s lignes comprenant des termes dominants en $\lambda_{s+1}^{N}, \cdots \cdot \lambda_{r}^{N}$.

A. la limite $\mathbb{N} \rightarrow \infty$, l'équation qui définit les modes de frontière se réduira, pour $|\rho|>I$, à la forme

$\{\operatorname{det}(I)\} \times\{\operatorname{det}(I V)\}=0$

Les racines $p$ seront donc obtenues en considérant séparément les équations 
$\operatorname{det}(I)=0$

$\operatorname{det}(I V)=0$

La première ne fait intervenir que les conditions aux limites en $x=x_{0}$ et la seconde les conditions aux limites en $x=x$. C'est la propriêté de découplage annoncée. De plus, il est clair que les colonnes du déterminant (IV) possèdent des facteurs communs $\lambda_{s+1}^{N}, \ldots . \lambda_{r}^{N} q u i$ peuvent être sup.. primés (car les $\lambda$ sont de module supérieur a l'unité, donc différents de zéro). Il en résulte que le nombre de mailies N n'apparâtra plus dans la seconde equation, de sorte que le nombre de solutions possibles de l'equation en $\rho$ est indépendant de $N$ pour $|\rho|>I$.

Nous pouvons done maintenant définir, du moins pour $N \rightarrow \infty$ * des modes instables de frontière pour la frontière $x=x_{0}$ et des modes instables de frontière pour la frontière $x=X$. Les premiers seront de la forme

$u_{j}^{k}=\rho^{k}\left(A_{1} \lambda_{1}^{j}+\ldots+A_{s} \lambda_{s}^{j}\right)$

oì

$|\rho|>1,\left|\lambda_{1}\right|<1, \ldots\left|\lambda_{s}\right|<1$

Un système linéaire homogène de s équations pour les $s$ inconnues $A_{1}$..... A s s'obtiendra en exprimant que les s conditions homogènes imposées à la frontière $x=x_{0}$ sont satisfaits. Une telle solution non triviale ne peut exister que si l'équation (3.2) est satisfoite. Cette équation fournira les valeurs possibles de $\rho$ et $\lambda$.

L'existence de tels modes n'est pourtant pas garantie. puisque les valeurs de $\rho$ et $\lambda$ ainsi obtenues ne satisfont pas nécessairenent les inégalités (3.5).

En fait, La conaition nécessaire qui résulte de cette analyse est tout simplement la suivante: Il ne peut pas exister de mode instable à la frontière compte tenu des conditions aux limites appliquées. C'est Ia condition de Godunov-Ryabenkii.

La définition des modes instables à la frontière $x=X$ est tout à fait analogue, les mođules des $\lambda$ étant, cette fois, supérieurs à l'unité.

Avant d"uliustrer ces developpements par des exemples choisis pour leur simplicité, il ne semble pas inutile d"insister sur les 
difficultés algébriques posées par l'étude des modes instables de frontière dans les applications pratiques.

Le fait que l'équation algébrique en $\rho$, dont le degré crồt avec $N$, se réduise pour les modes instables à deux équations de degrés très limités (s et $r-s$ ) représente, certes, une simplification très spectaculaire.

Néanmoins, la discussion paramétrique, dans le plan complexe, de solutions d'êquations algébriques, même de degré relativement bas, en présence de conditions auxiliaires de type (3.5), présente souvent des difficultês nettement plus considérables que la mise en oeuvre du critère de von Neumann. Cette discussion nécessite souvent l'utilisation d'un ordinateur et il y a lieu de craindre qu'elle ne conduise, dans certains cas, à des temps de calcul non négligeables par rapport au temps de calcul requis par la solution de l'équation aux dérivées partielles.

\section{Exemple d'instabilité induite par des conditions aux limites naturelies}

Considérons le systèmes hyperbolique du second ordre pour les deux fonctions inconnues $u, v$ des variables $t, x$ :

$\frac{\partial u}{\partial t}+\frac{\partial v}{\partial x}=0 \quad \frac{\partial v}{\partial t}+\frac{\partial u}{\partial x}=0$

Ce système est équivalent à l'équation normalisée des ondes dans un espace à une dimension.

Les conditions aux limites seront du type classique :

u (ou v) donné aux frontières $x=x_{0}$ et $x=x \quad$ (4.2)

Une discrétisation du premier oràre de précision peut être obtenue par l'artifice suivant : deux combinaisons linéaires indépendantes des équations (4.1) sont formées à l'aide de deux constantes distinctes $a, B$ :

$\frac{\partial(u+\alpha v)}{\partial t}+\frac{\partial(v+\alpha u)}{\partial x}=0 \quad \frac{\partial(u+\beta v)}{\partial t}+\frac{\partial(v+\beta u)}{\partial x}=0$

Les dérivées par rapport à t sont discrétisées en utilisant des différences avant. La dérivée par rapport à $x$ dans la première équation sera discrétisée en utilisant également des différences avant, mais des différences arrières seront utilisées pour la discrétisation, 
selon $x$, de $2 a$ seconde équation. Le schéma de discrétisation ainsi obtenu s'écrit donc :

$u_{j}^{k+1}-u_{j}^{k}+a\left(v_{j}^{k+1}-v_{j}^{k}\right)+c\left\{v_{j+1}^{k}-v_{j}^{k}+a\left(u_{j+1}^{k}-u_{j}^{k}\right)\right\}=0 \quad(4,3)$
$u_{j}^{k+1}-u_{j}^{k}+s\left(v_{j}^{k+1}-v_{j}^{k}\right)+c\left(v_{j}^{k}-v_{j-1}^{k}+\beta\left(u_{j}^{k}-u_{j-1}^{k}\right)\right\}=0 \quad(4 \cdot 4)$

où c désigne le nombre de Courant

$c=\frac{\Delta t}{\Delta x}$

La généralisation de la méthode de separation des variables au cas des systèmes discrétisés est immédiate et consiste à étudier des solutions de la forme

$u_{j}^{k}=u_{k} \lambda^{j}, \quad v_{j}^{k}=v_{k} \lambda^{j}$

Introduisant ces expressions dans les équations discrétisées et en les résolvant par rapport $\bar{a} U_{k+1}$ et $V_{k+1}$, nous obtiendrons

$U_{k+1}=\left\{1+\frac{\alpha \beta c}{\alpha-\beta}\left(\lambda+\frac{1}{\lambda}-2\right)\right\} U_{k}+c \frac{\beta \lambda+\frac{\alpha}{\lambda}-(\alpha+\beta)}{\alpha-\beta} v_{k}$

$v_{k+1}=c \frac{\alpha+\beta-\alpha \lambda-\frac{\beta}{\lambda}}{\alpha-\beta} U_{k}+\left\{1+\frac{c}{\alpha-\beta}\left(2-\lambda-\frac{1}{\lambda}\right)\right\} v_{k}$

La matrice d"amplification est donc donnêe par

$$
\left[\begin{array}{ll}
1+\frac{\alpha \beta c}{\alpha-\beta}\left(\lambda+\frac{1}{\lambda}-2\right) & c \frac{\beta \lambda+\frac{\alpha}{\lambda}-(\alpha+\beta)}{\alpha-\beta} \\
c \frac{\alpha+\beta-\alpha \lambda-\frac{\beta}{\lambda}}{\alpha-\beta} & I+\frac{c}{\alpha-\beta}\left(2-\lambda-\frac{1}{\lambda}\right)
\end{array}\right]
$$

Les valeurs propres $\rho$ de cette matrice satisfont l'équation caractéristique du schéma $(4.3),(4.4):$

$$
\left|\begin{array}{ll}
1-p+\frac{\alpha \beta c}{\alpha-\beta}\left(\lambda+\frac{1}{\lambda}-2\right) & c \frac{\beta \lambda+\frac{\alpha}{\lambda}-(\alpha+\beta)}{\alpha-\beta} \\
c \frac{\alpha+\beta-\alpha \lambda-\frac{\beta}{\lambda}}{\alpha-\beta} & 1-p+\frac{c}{\alpha-\beta}\left(2-\lambda-\frac{1}{\lambda}\right)
\end{array}\right|=0
$$


Après quelques réductions, cette équation s'écrit:

$F(0, \lambda)=(1-0)^{2}+c \zeta\left(\lambda+\frac{1}{\lambda}-2\right)(1-p)-c^{2}\left(\lambda+\frac{1}{\lambda}-2\right)=0 \quad(4.5)$

en posant

$\zeta=\frac{\alpha \beta-1}{\alpha-\beta}$

Remarquons que cette équation est réciproque en $\lambda$ de sorte qu'elie possède pour toute valeur de $\rho$ deux racines $\lambda_{1}, \lambda_{2}$ telles que

$\lambda_{1} \lambda_{2}=1$

Si nous prenons, pour $\lambda$, des valeurs exponentielles complexes du type (2.2), la relation caractéristique s'écrira

$(1-\rho)^{2}-2 c \zeta(1-\cos \theta)(1-\rho)+2 c^{2}(1-\cos \theta)=0$

en posant

$\theta=i \pi \frac{n}{N} \quad(n=0,1, \ldots N)$

Le critère de stabilité de von Neumann exige que le modules des racines $p$ de l'équation $(4.7)$ soient inférieures à l'unité quel que soit $\theta$.

Il faut donc que le produit de ces modules soit inférieur à I'unité :

$\left|1-2 \cos (1-\cos \theta)+2 c^{2}(1-\cos \theta)\right| \leq 1$

Cette condition doit être satisfaite pour toutes valeurs réelles de $\theta$, ce qui implique

$\frac{1}{2} \geq c(\zeta-c) \geq 0$

Il faut également que le premier membre de (4.7) soit non négatif pour $\rho=+1$ et $\rho=-1$, d'où la nouvelle condition

$4-4 \cos (1-\cos \theta)+2 c^{2}(1-\cos \theta) \geq 0$

pour toute valeur rélle de $\theta$. Nous devons donc avoir 


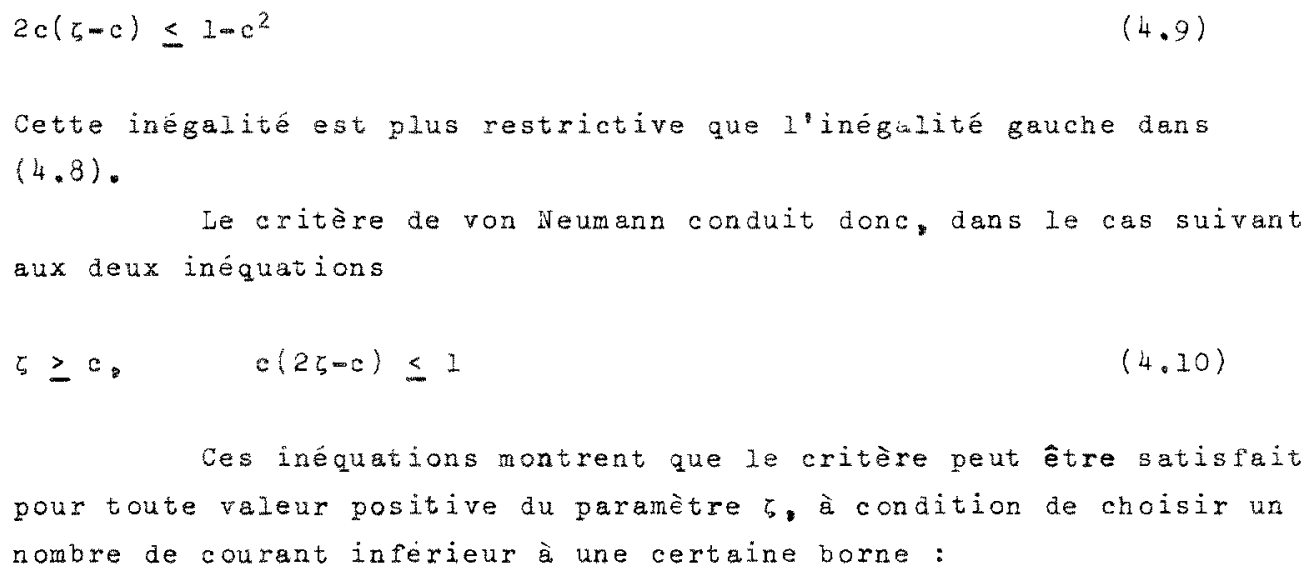

Ces inéquations montrent que le critère peut être satisfait pour toute valeur positive du paramètre $\zeta$, à condition de choisir un nombre de courant inférieur à une certaine borne :

Considérons maintenant les modes de frontières instables éventuels qui pourraient résulter de l'application d'une condition naturelle du type $(4.2)$, par exemple:

$u(x, t)=f(t) \quad$ pour $x=x_{0}$

Les résultats s'appliqueront, évidement, moyennant modifications évidentes au cas ou $V$ serait impose en $x=x_{0}$. et aux conditions la frontière $x=x$. La condition homogene discrétisée correspondant à $(4.12)$ s'écrit

$u_{0}^{k}=0 \quad$ pour $k=1,2, \ldots$

Pour toute valeur de $\rho$ de module supérieur a l'unite, il existera. en vertu de (4.6), deux racines $\lambda_{1}, \lambda_{2}$ de la relation caractéristique telles que

$\left|\lambda_{1}\right|<1<\left|\lambda_{2}\right|$

Seul le mode correspondant à $\lambda_{1}$ pourra être considéré comme mode de la frontière $x=x_{0}$. en accord avec les conclusions de la section 3 . Ce mode s'écrira

$u_{j}^{k}=u_{1} \rho^{k} \lambda_{1}^{j} \quad v_{j}^{k}=v_{1} \rho^{k} \lambda \lambda_{1}^{j}$ 
et la condition à la limite (4.13) imposera

$\mathrm{U}_{1}=0$

L'amplitude $V_{1}$ peut être obtenue à partir de l'une ou l'autre des équations aux différences finies (4.3), (4.4) (qui sont équivalentes, du fait que $\rho, \lambda_{1}$ sont liées par la relation caractéristique). Nous avons, par exemple,

$\left\{\alpha(p-1)+c\left(\lambda_{1}-1\right)\right\} \quad v_{1}=0$

Un mode non trivial ne peut donc exister que si la condition suivante est satisfaite :

$\alpha(0-1)+c\left(\lambda_{1}-1\right)=0$

Combinant cette relation avec la relation caractéristique (4.5), nous obtiendrons

$\lambda_{1}=\frac{\alpha}{\beta}, \quad \rho=1-c\left(\frac{1}{\beta}-\frac{1}{\alpha}\right)$

Ces valeurs définiront donc un mode instable pour la frontière $x=x_{0}$ à condition que

$\left|\frac{\alpha}{\beta}\right|<1, \quad\left|2-\operatorname{co}\left(\frac{1}{\beta}-\frac{1}{\alpha}\right)\right|>1$

Une discussion complète de ces conditions peut être effectuée dans le plan $\alpha, \beta$. Nous nous contenterons de démontrer ici qu'un tel mode instable peut exister; même si le critère de von Neumann, exprimé par (4.11), est satisfait. En effet, considérons le cas où

$\alpha=\frac{1}{2}, \quad \beta=1 \quad$ d'où $\quad \zeta=1$

La condition de von Neumann sera flors satisfaite si

$c=\frac{\Delta t}{\Delta x} \leq c_{m}(I)=I$

mais il existera un mode de frontière instable avec $\lambda=\frac{1}{2}, \rho=1+c$ : $u_{j}^{k}=0 \quad v_{j}^{k}=$ const $\cdot(I+c)^{k} 2^{-j}$ 
Cet exemple d'instabilité est évidement surprenant puisqu'il ne peut pas être attribué au choix malencontreux d'une condition aux limites aditionnelles plu's ou moins arbitraire. Les conditions au type (4.12) sont en effet tout à fait naturelles en physique et conduisent toujours à des problèmes hyperboliques bien posés.

\section{Instabilités causées par des conditions adaitionnelies}

Les exemples d'instabilités résultant dun choix malencontreux des conditions aux limites adjitionnelies, requises par les discrétisations d'ordre de précision supérieur au premier, sont très nombreux et il importe donc d'attirer l'attention sur cette situation dangereuse. Nous allons donner ici un exemple très simple qui indique sans nécessiter de longs calculs la grande variété des choix "raisonnables" de conditions aux limites additionnelles qui peuvent conduire à des instabilites.

A cet effet, nous examinerons l'équation hyperbolique la plus simple:

$\frac{\partial u}{\partial t}=\frac{\partial u}{\partial x}$

que nous discrétiserons à l'aide du schéma du second ordre de précisoin proposé par Lax et Wendroff (ref. 2 et 7 ):

$u_{j}^{k+1}-u_{j}^{k}=\frac{c}{2}\left(u_{j+1}^{k}-u_{j-1}^{k}\right)+\frac{c}{2}\left(u_{j+1}^{k}+u_{j-1}^{k}-2 u_{j}^{k}\right)$

c désigne le nombre de Courant $\frac{\Delta t}{\Delta x}$.

On sait que ce schema est stable au sens de von Neumann. pour $c \leq 1$, ce qui se vérifie d'ailleurs aisément en se reférant à Ia relation caractéristique

$0-1=\frac{c}{2}\left(\lambda-\frac{1}{\lambda}\right)+\frac{c^{2}}{2}\left(\lambda+\frac{1}{\lambda}-2\right)=\frac{c}{2} \frac{\lambda-1}{\lambda}((1+c) \lambda+1-c)$

Les conditions initiales et aux limites naturelles pour le problème hyperbolique ans l'intervalle spatial

$x_{0} \leq x \leq x$

consistent à imposer u pour $t=t_{0}$ et pour $x=X$, puisque les caractéristiques $(x+t=$ const) de I'équation (5.1) sont orientées vers les $x$ décroissant pour $t$ croissant. 
Cependant, comme l'équation aux différences (5.2) fait intervenir des différences centrees, le processus numérique de prograssion ne sera défini, dans l'intervalle $\left(x_{0}, x\right)$, que si l'on impose une condition additionnelle numerique en $x=x_{0}$.

Nous allons considerer des conditions de deux types différents qui forment un ensemble assez général. Le premier type fait intervenir les valeurs de u aux quatre points $(k, 0),(k, 1),(k+1,0)$, $(k+1,1)$ et seront de la forme

$u_{0}^{k+1}+\ell u_{1}^{k+1}+m u_{0}^{k}+n u_{1}^{k}=0$

En se servant de la relation $(5.2)$ on peut a'ailleurs eliminer $u_{1}^{k+1}$ et exprimer la valeur inconnue $u_{0}^{k+1}$ en fonction des valeurs $u_{0}, u_{1}$ et $u_{2}$ à 1 'étape $k$.

Le secona type de conaitions considéré ne fait intervenir que des valeurs à l'instant $x+1$, et ces conditions seront de 1 a forme

$u_{0}^{k+1}+f u_{1}^{k+1}+g u_{2}^{k+1}=0$

De telles conditions additionnelles ne peuvent pas introduire des erreurs $O(1)$, ce qui détruirait la précision du calcul, mais elles deviennent compatibles à l'ordre $O(\Delta x, \Delta t)$ à condition que les sommes des coefficients des u soient nulles:

$1+\ell+m+n=0 \quad I+f+g=0$

Appliquons maintenant la théorie générale de la section 3 . II ne peut $y$ avoir au plus qu'un mode instable pour la frontière $x=x_{0}$. Soit $\lambda$, la valeur correspondante de

$\left|\lambda_{1}\right|<1$

Nous allons montrer que les valeurs de $\rho$ et $\lambda_{1}$ qui permettent de satisfaire les conditions (5.5) sont réelles et que cela est également vrai pour les conditions $(5.4)$ si la précision qutelle fournit est du second orare ${ }^{1}$.

Il faut noter que cette précision n'est pas strictement nécessaire pour obtenir un résultat précis du second ordre. 
En effet, écrivons les conditions $(5.4)$ et $(5.5)$ en y introduisant $I^{\prime}$ expression du mode instable éventuel

$u_{j}^{k}=\operatorname{const} \rho^{k} \lambda_{1}^{j}$

Nous obtiendrons, respectivement,

$\rho+\ell \lambda \rho+m+n \lambda=0$

et

$1+f \lambda+g \lambda^{2}=0$

Si nous exprimons maintenant $\rho$ en ponetion de $\lambda$, dans (5.7), en utilisant la relation caractéristique (5.3) du schéma, nous obtiendrons une équation du 3me degré en $\lambda$ :

$a_{3} \lambda_{1}^{3}+a_{2} \lambda_{1}^{2}+a_{1} \lambda_{1}+a_{0}=0$

En vertu des conditions de compatibilité (5.6). les équations $\left(5.7^{\prime}\right)$ et (5.8) possèderont une racine $\lambda$, égale à l'unité, qui correspond d'ailleurs au mode trivial $0=1$ que l'on peut écarter. Si nous exigeons, de plus, que la condition (5.4) présente une précision du se-

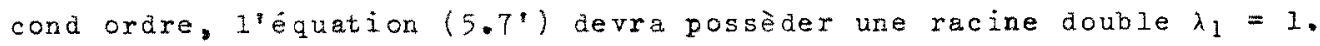
Après élimination des facteurs $\left(\lambda_{1}-1\right)$ dans l'équations $(5.8)$ et $\left(\lambda_{1}-1\right)^{2}$ dans $1^{\prime e ́ q u a t i o n ~\left(5.7^{\prime}\right)}$ nous obtiendrons, dans les deux cas, une equation du premier ordre en $\lambda_{1}$ :

$p \lambda_{1}+q=0$

dont la racine - $\frac{q}{p}$ est toujours réelle.

Il est facile de vérifier que le rapport $\frac{g}{g}$ sera une fonction $r$ ationnelie non constante des paramètres $l, m, n, f, g$ qui définissent les conditions aditionnellees considérés. Il existera done toujours des valeurs de ces paramètres pour lesquelies

$\left|\lambda_{1}\right|=\left|\frac{p}{q}\right|<1$

$\lambda_{1}$ étant réel, il en sera de même pour la valeur corresponante de tirée de (5.3). Nous aurons donc un mode instable si l'une des deux inéqualités suivantes est vérifiée: 
$p=1+\frac{c}{2} \frac{\lambda_{1}-1}{\lambda_{1}}\left((1+c) \lambda_{1}+1-c\right) \quad>\quad 1 \quad$ ou

Il est facile de vérifier que l'une de ces conditions sera satisfaite (compte tenu de $\left|\lambda_{1}\right|<1$ ), si

$-1<-\frac{1-c}{1+c}<\lambda_{1}=-\frac{p}{q}<\frac{c^{2}-2+\sqrt{4-3 c^{2}}}{c(c+1)}<1$

(Les inégalités extrêmes résultent de la condition de Courant $0<c<1$ ). Nous n'entrerons pas dans les détails d'une discussion complète car les inégalités (5.9) suffisent à montrer qu'il existera toujours des valeurs "raisonnables" des paramètres $l, m, n, f, g$ pour lesquelles l'inégalité (5.9) pourra être satisfaite et qui conduiront donc à des instabilités, malgré le fait que le critère de von Neumann est respecté.

\section{Modes de frontière non exponentiels}

Kreiss a signalé (réf. 6) que l'absence de mode instable de frontière du type introduit à la section 3 n'est pas suffisante pour assurer la stabilité. Il a pu, en effet, donner un exemple de solution croissante avec $t$, mais non exponentielle et a égelement établi un critère plus restrictif que celui de Godunov-Ryabenkii, qui tient compte de l'existence de telles solutions.

Un traitement numérique de l'exemple propose par Kreiss fait apparaitre certaines propriêtés remarquables de ces solutions croissantes et nous nous proposons de clarifier leur nature en utilisant ces propriétés.

L'équation hyperbolique considérée est l'équation simple (5.I) traitée dans le domaine $t \geq 0, x_{0} \leq x \leq x$.

L'exemple de Kreiss fait appel au schéma aux différences symétrique ("Leap frog")

$u_{j}^{k+1}-u_{j}^{k-1}=c\left(u_{j+1}^{k}-u_{j-1}^{k}\right) ; \quad c=\frac{\Delta t}{\Delta x}$

stable au sens de ron Neumann si la condition de Courant $(0<1)$ est vérifiée.

Il est important de remarquer que ce schéma fait intervenir trois niveaux temporels $k+1$, $k$ et $k-l$; contrairement au schema de LaxWendrofe utilisé à la section 5. Cette propriëté joue un rôle essentiel dans notre interprétation des solutions de Kreiss. 
Le schema $(6.1)$ étant du second ordre de precision par rapport à $x$, il faudra comme précédemment, ajouter à la condition naturelle en $x=x$, une condition additionnelle en $x=x_{0}$.

Nous examinerons les con aitions suivantes, dont certaines ont été proposees par Kreiss (rég. 6) et qui dont toutes compatibles à l'ordre $(\Delta t, \Delta x)$ au moins:

(I) Extrapolation linéaire: $u_{0}^{k}=2 u_{1}^{k}-u_{2}^{k}$

(II) Extrapolation modifiée proposée par Kreiss $=u_{0}^{k}=\left(u_{1}^{k-I}+u_{1}^{k+I}\right)-u_{2}^{k}$ $\left(u_{1}^{k+1}\right.$ est calculé en fonction de $u_{0}^{k}$ et $u_{2}^{k}$ à partir de (6.1))

(III) Extrapolation parabolique $u_{0}^{k}=3 u_{1}^{k}-3 u_{2}^{k}+u_{3}^{k}$

(IV) Interpolation d'orare zéro: $u_{0}^{k}=u_{1}^{k}$.

Montrons tout a'abord qu'il n'existe pas de mode instable de frontière au sens de la section 3 . La relation caractéristique du schéma (6.1) s'écrit:

$\rho-\frac{1}{\rho}=c\left(\lambda-\frac{1}{\lambda}\right)$

Il n'existe, comme prévu, qu'un mode instable de frontière possible puisque le produit des racines de (6.2) vaut - L. Les conditions aux limites (I à IV) fournissent.respectivement les équations suivantes pour $\lambda_{1}$ :

$\left(\lambda_{1}-1\right)^{2}=0 ; \quad \lambda_{i}+\frac{1}{\lambda_{1}}=0+\frac{1}{0} ; \quad\left(\lambda_{1}-1\right)^{3}=0 ; \quad\left(\lambda_{1}-1\right)=0$

Ces relations conduisent toutes à des valeurs de $\lambda$ et $p$ dont le module est égal à $I^{\prime}$ unité, ce qui suggère une stabilité marginale en présence de ces conditions additionnelles.

Kreiss signale cependant que la condition (I) introduit des solutions croissant au delà de toute limite aveck, et que ce phénomène disparastt lorsque $I^{\prime}$ on utilise la forme légèrement modifiée (II). Le calcul numérique d'un exemple basé sur la condition (I)

fait immédiatement apparâtre les faits suivants: Après disparition de contributions transitoires amorties introduites par les conditions initiales numériques particulières, la solution présente un comportement alterné par rapport au temps :

$u_{j}^{k+1}=-u_{j}^{k}$

et la dépendance de $u_{j}^{k}$ par rapport à $x$ est très voisine a'une loi 1 inéaire. Il en est d'ailleurs de même pour la dépendance en fonction de $t$, des valeurs bolues $\left|u_{j}^{k}\right|$. 
Les fonctions discrètes $u_{j}^{2 k}$ et $u_{j}^{2 k+1}$ apparaissent donc comme essentiellement distinctes et ne peuvent, en vertu de (6.3), être considérés comme formant la représentation discrète d'une fonction dérivable, ni même continue de t. Cependent, si nous posons

$u_{j}^{2 \ell}=v_{j}^{\ell}, \quad u_{j}^{2 \ell+1}=w_{j}^{\ell}$,

nous pourrons considérer $v$ et $w$ comme représentation discrète de deux fonctions distinctes $v$ et $w$, continues et même dérivables.

Le schéma aux différences finies $(6.1)$ écrit pour $k=2 \ell$ et $k=2 \ell+1$, peut être considéré comme formant un système de deux équations aux différences couplées pour v et $w$ :

$w_{j}^{\ell}-w_{j}^{\ell-1}=c\left(v_{j+1}^{\ell}-v_{j-1}^{\ell}\right)$

$v_{j}^{\ell+1}-v_{j}^{\ell}=c\left(w_{j+1}^{2}-w_{j-1}^{\ell}\right)$

Nous considèrerons maintenant ces équations comme des discrétisations du système hyperbolique

$\frac{\partial w}{\partial t}=\frac{\partial v}{\partial x}, \quad \frac{\partial v}{\partial t}=\frac{\partial w}{\partial x}$

La solution générale de ce système est donnée par

$v=F(x-t)+G(x+t) ; \quad w=-F(x-t)+G(x+t)$

$(6.7)$

Il est clair que la contribution G représente la solution générale de l'équation de départ (5.1), tandis que F représente une contribution parasite introduite par la discrétisation d'ordre supérieur. En effet, si $F \equiv 0$, nous avons

$v=w=G(x+t)=u$

La contribution parasite $F$ correspond à des caractéristiques parasites $x-t=$ const, introduites par le système $(6,6)$ et est la cause de l'instabilité signalée par Kreiss.

Si la condition initiale est homogène ( $u=0$ pour $\left.t=t_{0}\right)$, seule la contribution parasite sera prêsente, comme on le voit facilement en tenant compte de l'orientation des caractéristiques naturelles $(x+t=$ const $)$ et parasites $(x-t=$ const $)$.

Les conditions aux limites (I) à (IV) seront, elles ausi 
interprétées comme des relations entre $v$, et leurs dérivées et nous aurons ainsi, respectivement

aurons ainsi, respect $\frac{\partial^{2}}{\partial x^{2}}$ (I) $\frac{\partial^{2} y}{\partial x^{2}}=0 \quad \frac{\partial^{2}}{\partial x^{2}}=0$

(II $I^{8} \quad \mathrm{v}=\mathrm{w}$

(III') $\frac{\partial^{3} v}{\partial x^{3}}=0 \quad \frac{\partial^{3} w}{\partial x^{3}}=0$

(IV $\left.V^{2}\right) \frac{\partial V}{\partial x}=0 \quad \frac{\partial W}{\partial x}=0$

Il suffit de substituer dans ces relations les expressions (6.7) après avoir posé $G \equiv 0$. ce qui donne les équations suivantes pour la fonction $F$ qui représente la contribution parasite:

$\begin{array}{ll}\text { (I") } F^{\prime \prime}\left(x_{0}-t\right)=0 & \text { d'où } F=A(x-t)+\text { const } \\ \text { (II") } F\left(x_{0}-t\right)=0 & \text { d'où } F=B(x-t)^{2}+C(x-t)+\text { const } \\ \text { (III") } F^{\prime \prime}\left(x_{0}-t\right)=0 & \text { d'où } F=\text { const } \\ \text { (IV") } F^{\prime}\left(x_{0}-t\right)=0 & \text { d }\end{array}$

La croissance Iineaire de la perturbation en fonction de $x$ et $t$ apparait donc clairement pour le première condition, alors que cette perturbation est étouffée par la condition modifiée (II). La condition (III) montre que des croissances suivant nimporte quelle puissance entière de $t$ sont possibles si l'on utilise des extrapolations d'ordre élevé. Par contre, la perturbation reste stationnaire dans le cas de l'extrapolation d'ordre zéro.

Les condidérations qui précèdent ne sont évidemment pas rigoureuses et ne semblent s'appliquer que s'il existe un découplage entre certaines lignes de maillage, qui semble être une caractéristique des schémas de type "leap fog".

Tenant compte de l'existence de cette nouvelle classe d'instabilité, Kreiss a puétablir une condition suffisante de stabilité (réf. 6) qui requiert que le premier membre de l'équation aux valeurs propres $p_{v}$, introduite à la section 3 , reste supérieur à une constante positive, quel que soit le nombre complexe p de module supérieur à l'unite. Cette condition exprime, en fait, qu'il ne doit pas exister de mode de frontière marginalement stable. 
Les solutions à croissance algébriques, en

$k^{n}=\left(\frac{t}{\Delta t}\right)^{n}$

qui viennent d'être discutées, sont en principe moins génantes que les instabilités exponentielles, et pourraient même être acceptables dans les cas où le maillage temporel utilisé est relativement grossier.

\section{Conclusions}

Le but de la présente étude était de montrer la variété des instabilités numériques qui peuvent être introduites dans les problèmes hyperboliques par les conditions aux limites.

Notre premier exemple a montré que des conditions aux limites tant à fait naturelles pouvaient conduire à une restriction de la stabilité par rapport au critère de von Neumann.

Les exemples d'instabilités provoqués par l'introduction de conditions numériques additionnelles sont très nombreux. Signalons d'ailleurs que des conditions construites à partir d'extrapolations, couramment utilisées, peuvent conduire à des instabilités si leur ordre est trop élevé, dans le cas de systèmes hyperboliques. Moretti (réf. 4) a préconisé l'utilisation de conditions additionnelles basées sur des considérations physiques inspirées de la thếorie des caractéristiques.

Une telle approche semble donner des résultats favorables ans la plupart des exemples traités, mais il n'existe pas, à notre connaissance, de preuve rigoureuse de son efficacité. II ne faut d'ailleurs pas perdre de vue la nature essentiellement numerique des conaitions additionnelles ce qui ne permet pas d'espérer, a priori, que des considérations physiques soient un bon guide pour leur sélection.

Notre manque d'informations générales permettant d'orienter de façon sure, le choix des conditions additionnelles, résulte évidemment de la grande complexité algébrique présentée par la mise en ouvre des cxitères de Godunov-Ryabenkii et de Kreiss, pour les provlèmes hyperboliques non triviaux rencontrés en pratique.

Nous nous sommes linités aux problèmes à une seule dimension spatiale afin de rendre le traitement algébrique suffisament accessible. L'extension des résultats et méthodes aux problèmes à plusieurs dimensions spatiales ne présente pas de difficultés fondamentales. Par exemple, si une condition est donnée, dans un cas bidimensionnel, sur Ia ligne $x=x_{0}$, il faudra introduire des modes du type 
$u_{j 2}^{k}=\operatorname{const} o^{k} \lambda^{j} j^{2}$

ou I'indice \& représente 1 a dépendance par rapport à $y$ Nous pouvons toujours représenter une telle dépendance par des modes de Fourier du maillage en $y$ et poserons donc

$\mu=e^{\dot{i \theta}}$

L"introduction du nouveau paramètre $\theta$ dans $7 a$ aiscussion de la stabilite est évidemment de nature à créer des complications additionnelies.

Enfin, des méthodes analogues pourraient être exploitées pour I'étude des instabilités qui apparaissent dans les "coins" de domaines multidimensionnels. Il s'agirait, par exemple, d'étudier le comportement atune solution numérique d'un problème d'écoulement bidimensionnel dans une conduite, au voisinage du point de reacontre entre une paroi et une ligne portant les conditions amont ou aval. Nous aurions, par exemple, a traiter une condition (A) à la frontière $x=x_{0}, y>y_{0}$ et une condition différente (B) à la frontière y $=y_{0}$. $x>x_{0}$. Il faudrat elors introduire des "modes de coin" fonctions exponentielles décroissantes de $x$ et $y$.

Les modes de Kreiss, étudiés à la section 6 , n'apparaissent vraisemblablement que dans des cas marginaux qui ne se manifesteront que pour des discrétisations particulières.

\section{Ré fó rences}

1. $0^{\circ} B R I E N, G, G$, HYMAN, M.A. et KAPLAN, S.: A study of the numerical solution of partial differential equations. J. Mathematios and Physics, vol. 29, 1950, pp 223-251.

2. RICHTMYER, R.D. et MORTON, K. W.: Difference methods for initial value problems. Interscience, 1967. New York.

3. KREISS, H.0.: On difference approximations of the dissipative type for nyperbolic differential equations. Comm. Pure Appl. Math. VoI. 17. 1964, p. 335.

4. MORETTI, G.: The importance of boundary conditions in the numerical treatment of hyperbolic equations. Brookzyn Polytechnic Institute, PIBAL Report 68-34.

5. GODUNOV, S.K. et RYABERKII. V.S.: Critères speciaux de stabilité des problèmes è conditions aux limites pour les équations 
aux différences finjes non auto-adjointes. Uspekhi Mat. Nauk., Vol. $18,1963, \mathrm{p} .3$

6. KREISS, H.O.: BOundary conditions for difference approximation of hyperbolic differential equations. in Advances in numerical fluid dynamics, AGARD Lecture Series No 64, AGARD, Neuillysur-Seine, France.

7. LAX, P.D. et WENDROFF, B.: Difference schemes for hyperbolic equations with high order of accuracy. Comm. Pure Appl. Math. Vol. 17, 1964, p. 381. 